\title{
Breastfeeding problems: an analysis of secondary data from Lactation Management Unit at the national referral hospital in Bhutan from 2014 to 2016
}

\author{
Kencho Zangmo ${ }^{1}$, Diki Wangmo ${ }^{2}$, Tashi Tobgay ${ }^{3}$, Mongal S Gurung ${ }^{4}$
}

\author{
'Jigme Dorji Wangchuck National Referral Hospital, Thimphu, Bhutan \\ ${ }^{2,3}$ Khesar Gyalpo University of Medical Sciences of Bhutan, Thimphu, Bhutan \\ ${ }^{4}$ Ministry of Health, Thimphu, Bhutan
}

\begin{abstract}
Introduction: Breastfeeding is one of the most natural and best forms of preventive medicine for the newborns. Lack of optimal breastfeeding contributes to about over million preventable child deaths every year. Despite numerous benefits of breastfeeding and continuous efforts to improve breastfeeding practices, breastfeeding rates are far from optimal. Problems faced by lactating mothers while breastfeeding are main reason leading to non-exclusive breastfeeding or early termination of breastfeeding. This descriptive study is aimed at examining breastfeeding problems among women visiting Lactation Management Unit [LMU] at Bhutan's national referral hospital in Thimphu. Methods: Data of all 2751 women-infant pair visiting Lactation management unit of Jigme Dorji Wangchuck National Referral Hospital with breastfeeding problems were double entered into Epi Data (version 3.1). Using STATA, descriptive statistical analyses were carried to examine common breastfeeding problems among the lactating mothers. Exclusive breastfeeding rates were also examined among the group. Results: The commonest types of breastfeeding problem were nipple sore $(60.8 \%)$, incorrect breastfeeding techniques (12.9\%), and nipple problems $(8.2 \%)$. Teenage, first timers, caesarean delivery, preterm infants, underweight infants, and mothers with full time paid job were more likely to suffer from breastfeeding problems. Conclusions: Breastfeeding interventions for timely correction of breastfeeding techniques early in postnatal units could warrant prevention of up to $73.7 \%$ breastfeeding problems and thus, promoting exclusive breastfeeding and preventing early termination of breastfeeding.
\end{abstract}

Keywords: Breastfeeding problems; Breastfeeding techniques; Exclusive breastfeeding; Nipple sore.

\section{INTRODUCTION}

Breastfeeding is one of the most economic, natural, and best form of preventive medicine for the newborn. According to the World Health Organization (WHO), lives of over 820000 children could be saved every year if all under-two years children are optimally breastfed ${ }^{1}$. Similarly, the United Nations International Children's Emergency Fund (UNICEF) has estimated that almost 1.3 million children under the age of 5 could be saved annually through exclusive breastfeeding alone ${ }^{2}$. Despite of the numerous benefits of breastfeeding and continuous effort to improve breastfeeding practices, breastfeeding rates are far from optimal. Studies have suggested so many maternal, infant, socio-demographic, and support system factors related to low exclusive breastfeeding rates $^{3-5}$. The recent studies suggest that the problems faced by lactating mothers while breastfeeding might be main reason leading to non-exclusive breastfeeding or early termination of breastfeeding ${ }^{6-8}$. A study in India found out that $88.5 \%$ of

\section{Corresponding author:}

Kencho Zangmo

kenchozangmo84@gmail.com mothers faced problems related to breastfeeding, of which 70.8\% had positioning and attachment problems while 10.5\%-17.8\% suffered from nipple sore ${ }^{6}$. In addition, approximately $60 \%$ mothers were found to be terminating breastfeeding earlier than they desired because of the problems they faced in the process of breastfeeding 9 .

Although Bhutan has come long way in terms of promoting, supporting and sustaining breastfeeding practices, exclusive breastfeeding rate for under 6 months children is still low with only $48.7 \%$ of women exclusively breastfeeding their child $^{10}$. The same survey carried out in 2011 also reported that $11.5 \%$ of children aged $0-23$ months were receiving formula milk, suggesting early termination of breastfeeding. However, a little is known about there as on for low rates of exclusive breastfeeding and early termination of breastfeeding among Bhutanese children. A hospital based report (unpublished) from 2014 on problems of breastfeeding from the Lactation Management Unit (LMU) at JDWNRH showed $61 \%$ of the problems were sore nipple, $17 \%$ attachment problem, $8 \%$ engorged breast and blocked duct, and $11 \%$ were other problems such as inverted nipple, and flat nipple. However, to our knowledge no scientific study on breastfeeding problems in Bhutan has been done and published. 
Therefore, this study was carried out with the aim of providing some insight into the types of breastfeeding problems among the mother-infant pairs who visited LMU at JDWNRH.

\section{METHODS}

\section{Study design and setting}

This cross-sectional study used data of all 2751 women-infant pairs visiting LMU of JDWNRH from September 2014 to September 2016 with breastfeeding issues. Jigme Dorji Wangchuck National Referral Hospital (JDWNRH), the apex hospital in the country attends to about $41 \%$ of country's birth and had established a LMU in 2013 to attend to all lactating mothers with difficulty in breastfeeding. A register is maintained at LMU of JDWNRH to keep the record of patients visiting the unit. The register has basic information on both mother and infant such as their age, infant weight, gestational age, current breastfeeding status, and mode of delivery the register has recorded over 21 different types of breastfeeding problems.

\section{Ethical approval}

Ethical approval for this study and waiver for informed consent was sought from the Research Ethics Board of Health, Ministry of Health, Bhutan (REBH/Approval/2016/066). Site approval was sought from the hospital administrations, JDWNR hospital. All data collected were kept confidential and under no circumstances identifying characteristics of the participants were included in the study report. All data were anonymised with no identifiers to the patients.

\section{Data Management and Analyses}

Data were double entered and validated by the principal investigator from LMU register to Epi Data (version 3.1) with the help of an assistant reading it out from the register. Prior to data entry, visual checking and allotting serial number to all the cases were done. Statistical software STATA version IC/ 14 (licensed to Khesar Gyalpo Univeristy of Medical Sciences of Bhutan) was used for the data analysis. Descriptive statistics command such as frequency, percentage, mean, median, confidence interval, and standard deviation were used to describe the study variables. The LMU register recorded about 21 different types of breastfeeding problems and these problems were grouped as nipple sore, incorrect breastfeeding techniques, nipple problems, breast problem, perceived milk insufficiency or oversupply, and others. Each of these common problems were examined with sample characteristics such as maternal age, education, occupation, mode of delivery, infant gestational age and birth weight, number of LMU visits, and breastfeeding status of the infant. In addition, exclusive and non-exclusive breastfeeding rates were examined among women with different types of breastfeeding problems.

\section{RESULTS}

\section{Demographics}

During September 2014 to September 2016, a total of 9834 deliveries had occurred in JDWNRH, of which 8208 were normal and 1626 were caesarean section deliveries. Out of the total deliveries that had occurred in JDWNRH during the study period, approximately $28 \%$ of women had problems related to breastfeeding and they had visited LMU.

This study included all 2751 women-infant pair who had visited LMU with one or other problems of breastfeeding from September 2014 to September 2016. The number of visit to LMU ranged from 1-8 visits, with $73 \%$ of them making only 1 visit and $8 \%$ with more than 3 visits. Age of the mothers ranged from 14 to 46 years old with mean (SD) age of 26.2(4.7). This sample consisted of $61.4 \%$ housewives and rest with full time paid jobs. Majority of women had normal delivery $(86.8 \%)$ followed by $12.5 \%$ with caesarean delivery. Almost all the infants in the sample had normal birth weight $(92.9 \%)$. Fifty three percent of the infants were born term, $44 \%$ at post term, and $2.65 \%$ preterm.

\section{Common types of breastfeeding problems}

Women visiting LMU reported as many as 21 different problems that they faced while breastfeeding. These problems were grouped as: (1) nipple sore, (2) incorrect breastfeeding techniques (attachment, positioning, latching); (3) nipple problems (flat nipple, short nipple, inverted nipple, retracted nipple); (4) breast problems (engorgement, blocked duct, mastitis, breast abscess); (5) perceived milk insufficiency or over supply; and (6) others (pain while feeding, infant not suckling, lump in the breast, swollen lymph node, nipple confusion, maternal vomiting after feeding, and itching breast).

Majority of the women presented with nipple sore $(60.8 \%$, 95\% CI 58.9-62.6) followed by $13 \%$ percent (95\% CI $11.8-14.3$ ) with issues related to breastfeeding techniques (Table 1). Eight percent of the sample had nipple problem while breast problems and perceived insufficiency or over supply of breast milk were 7\% each. Teenage mothers, housewives, first time mothers, and mothers who had normal vaginal delivery, post term baby and overweight baby were more likely to present with nipple sore than their counterparts (Table 2). Mothers who had caesarean delivery and underweight babies faced issues related to breastfeeding techniques and those with preterm babies perceived milk insufficiency or oversupply more than all other groups.

\section{Exclusive breastfeeding practices}

Seventy two percent of infants were being exclusively breast fed around the time of their visit to LMU. The rates were $75.3 \%$ for 1 LMU visit and 59.2\% for more than 3 LMU visits (Table 3). Among the five main groups of breastfeeding problem, mothers with nipple problems and perceived breast milk insufficiency showed higher rates of non-exclusive breastfeeding. In addition, mothers who had caesarean and instrumental delivery did more non-exclusive $(55 \%$ \& $67 \%$ ) than exclusive breastfeeding ( $45 \%$ \& $33 \%)$. 
Table 1. Common types of breastfeeding problems among women visiting LMU

\begin{tabular}{lll}
\hline Common Breastfeeding Problems & Frequency & Percentage (95\%CI) \\
\hline Nipple sore & 1672 & $60.78(58.93-62.58)$ \\
Breastfeeding techniques & 357 & $12.98(11.77-14.28)$ \\
Nipple problems & 226 & $8.22(7.24-9.30)$ \\
Breast problems & 194 & $7.05(6.15-8.07)$ \\
Perceived milk insufficiency \& over supply & 202 & $7.34(6.42-8.37)$ \\
Others & 100 & $3.64(2.99-4.40)$ \\
Total & $\mathbf{2 7 5 1}$ & $\mathbf{1 0 0}$ \\
\hline
\end{tabular}

Table 2. Description of breastfeeding problems

\begin{tabular}{|c|c|c|c|c|c|c|c|}
\hline Variables & $\mathbf{N}$ & $\begin{array}{l}\text { Nipple sore } \\
(\%)\end{array}$ & $\begin{array}{l}\text { BFT } \\
(\%)\end{array}$ & $\begin{array}{l}\text { Nipple } \\
\text { problems } \\
\text { (\%) }\end{array}$ & $\begin{array}{l}\text { Breast } \\
\text { problems } \\
(\%)\end{array}$ & $\begin{array}{l}\text { PIOS } \\
(\%)\end{array}$ & $\begin{array}{l}\text { Others } \\
(\%)\end{array}$ \\
\hline Total & 2751 & 60.78 & 12.97 & 8.22 & 7.05 & 7.34 & 3.64 \\
\hline Teenage (<20yrs) & 151 & 60.93 & 9.93 & 15.23 & 5.30 & 4.64 & 3.97 \\
\hline Old age $(>35 \mathrm{yrs})$ & 123 & 50.41 & 13.82 & 8.13 & 8.94 & 13.01 & 5.69 \\
\hline Full time paid job & 1061 & 59.85 & 12.54 & 7.45 & 8.95 & 7.92 & 3.30 \\
\hline Housewives & 1690 & 61.36 & 13.25 & 8.70 & 5.86 & 6.98 & 3.85 \\
\hline Primipara & 1620 & 61.60 & 11.60 & 10.00 & 6.79 & 6.91 & 3.09 \\
\hline Multipara & 1131 & 59.59 & 14.94 & 5.66 & 7.43 & 8.96 & 4.42 \\
\hline Normal vaginal delivery & 2389 & 62.49 & 12.35 & 7.49 & 7.16 & 6.95 & 3.56 \\
\hline Caesarean delivery & 344 & 49.13 & 18.02 & 12.50 & 6.40 & 9.88 & 4.07 \\
\hline Underweight infant & 101 & 44.55 & 19.80 & 10.89 & 6.93 & 10.89 & 6.93 \\
\hline Normal weight infant & 2555 & 61.25 & 12.76 & 8.14 & 7.01 & 7.24 & 3.60 \\
\hline Overweight infant & 95 & 65.26 & 11.58 & 7.37 & 8.42 & 6.32 & 1.05 \\
\hline Preterm & 73 & 41.10 & 15.07 & 15.07 & 4.11 & 17.81 & 6.85 \\
\hline Term & 1458 & 58.02 & 13.31 & 8.50 & 7.48 & 8.23 & 4.46 \\
\hline Post term & 1220 & 65.25 & 12.46 & 7.46 & 6.72 & 5.66 & 2.46 \\
\hline
\end{tabular}

*BFT- breastfeeding techniques; PIOS- perceived milk insufficiency or oversupply.

Table 3. Description of exclusive and non-exclusive breastfeeding with other variables

\begin{tabular}{lcc}
\hline Variables & $\begin{array}{c}\text { Exclusive breastfeeding } \\
\boldsymbol{n}(\boldsymbol{\%})\end{array}$ & $\begin{array}{c}\text { Non-exclusive breastfeeding } \\
\boldsymbol{n}(\boldsymbol{\%})\end{array}$ \\
\hline Nipple sore & $1271(76.02)$ & $401(23.98)$ \\
Breastfeeding techniques & $260(72.83)$ & $97(27.17)$ \\
Nipple problems & $129(57.08)$ & $97(42.92)$ \\
Breast problems & $140(72.16)$ & $54(27.84)$ \\
Perceived milk insufficiency or oversupply & $116(57.43)$ & $86(42.57)$ \\
$\mathbf{1}$ LMU visit & $1523(75.27)$ & $497(24.73)$ \\
2 LMU visits & $355(69.20)$ & $158(30.80)$ \\
3 or more LMU visits & $135(59.21)$ & $93(40.79)$ \\
Normal vaginal delivery & $1841(77.06)$ & $548(22.94)$ \\
Caesarean delivery & $156(45.35)$ & $188(54.65)$ \\
Instrumental delivery & $6(33.33)$ & $12(66.67)$ \\
\hline
\end{tabular}




\section{DISCUSSION}

More than one-fourth of the women who delivered at JDWNRH between September 2014 and September 2016 had problems related to breastfeeding and they had visited LMU. The commonest type of breastfeeding problem among womeninfant pair visiting LMU, JDWNRH was nipple sore. The problems of breastfeeding can be related to one another in a very intricate way. Having one of those problems can lead to others in a form of progressive stages of a condition or present as a multiple problem at a time. However, the most common cause of nipple sore, which affects $61 \%$ of our lactating mothers, is incorrect breastfeeding techniques such as improper positioning and inappropriatelatching ${ }^{11,12}$. The problem with breastfeeding techniques can be seen early in postnatal period and this problem if not corrected in time led to nipple sore. The nipple sore further can progress to other problems of breastfeeding, ultimately affecting exclusive breastfeeding practices. Therefore, interventions to improve exclusive breast feeding should be aimed at correcting breastfeeding techniques early in postnatal period in addition to the already existing antenatal breastfeeding education services. This timely intervention could prevent $73.7 \%$ of breastfeeding issues all together.

Our findings on problems of breastfeeding were slightly different from what studies from Denmark and India have reported. An assessment of breastfeeding problems in postnatal mothers reported $88.5 \%$ with incorrect breastfeeding technique, $30.3 \%$ with nipple and breast problems, and $10.5 \%$ with sore nipple among Indian lactating mothers ${ }^{6}$. Incorrect breastfeeding techniques were also seen among 50\% of Danish mother-infant pairs, assessed early in postnatal period ${ }^{10}$. This difference could have resulted due to difference in timing of assessment of the problem. Other studies have assessed the problem early in postnatal period where initial breastfeeding issues such as incorrect breastfeeding techniques are commonly seen. On the other hand, our data consisted of variety of mother-infant pairs, ranging from few days to months old and thus, nipple sore, which occurs mainly from incorrect technique, was our commonest type of problem. These differences in finding also suggest the loophole in our maternity facilities to correct breastfeeding techniques at the right time; hence, majority of our women presented with sored nipples in later stage. Younger aged mothers presenting with more nipple sore, nipple problems, and perceived milk insufficiency or oversupply than their older counterparts point out the need for special attention in this group in breastfeeding interventions. Incorrect breastfeeding technique was a bigger problem among mothers who had caesarean and instrumental delivery than those who had normal delivery, in line with the findings from India which pointed out caesarean delivery as independent predictor of breastfeeding problem ${ }^{6}$.

Although, overall exclusive breastfeeding rate $(72.8 \%)$ is quite high in this sample, a notable decreasing pattern of exclusive breastfeeding was seen with the increase in number of LMU visits. This finding can be interpreted as women with breastfeeding problems are at higher risk for non-exclusive breastfeeding or early termination of breastfeeding. Our findings are in line with findings from international studies on problems of breastfeeding being one of the major contributors of nonexclusive breastfeeding or early termination of breastfeeding ${ }^{6}$.

9,11 . In fact, a study reported that breastfeeding problems gravely affected the standard breastfeeding practices as women with incorrect breastfeeding techniques are 4.42 times more likely to stop breastfeeding their infants ${ }^{9}$. Further, it was reported that overall $60 \%$ of women with breastfeeding problem stopped breastfeeding their infants earlier than they desired. Therefore, studying the problems of breastfeeding in depth and intervening at the right time could not only ensure exclusive breastfeeding and prevent early termination of breastfeeding but also save our mothers from chain of complications related to breastfeeding problems. The problem can start with a very simple and easily manageable issue as incorrect breastfeeding technique and end up with mastitis or breast abscess if not managed on time.

\section{Study limitation}

Since this study used secondary data from a register maintained at LMU, therefore the data were limited to only whatever was available in the register. Secondly, the findings can't be generalized to whole country as the data from only the national referral hospital was included in the study.

\section{CONCLUSIONS}

Nipple sore, which is the commonest type of breastfeeding problem among our sample suggest the dire need for timely correction of breast feeding techniques, the main cause of nipple sore and a breastfeeding problem by itself. Interventions to correct breastfeeding techniques doesn't cost much but little extra effort from maternity care providers in educating and aiding mothers with correct breastfeeding techniques early in postnatal period can make huge difference in improving breastfeeding practices. In addition, the existing antenatal breastfeeding education and services can be enhanced through timely correction of nipple problems and by enhancing women's knowledge and skill to breastfeed.

\section{REFERENCES}

1. World Health Organization (WHO). Media centre infant and young child feeding. WHO; 2016. [Full Text]

2. United Nations International Children's Emergency Fund (UNICEF). Breastfeeding within one hour of birth can reduce infant mortality. UNICEF; 2007. [Full Text] 
3. Lewis RB, Levkoff S, Stuebe A, Seely EW. Gestational diabetes mellitus: postpartum opportunities for the diagnosis and prevention of type 2 diabetes mellitus. Nat Clin Pract Endocrinal Metab 2008; 4(10): 552-58. [Full Text | DOI]

4. Borra C, Iacovou M, sevilla A. New evidence on breastfeeding and postpartum depression: the importance of understanding women's intentions. Matern Child Health J 2015; 19: 897-907.

5. National Cancer Institute. Ovarian, fallopian tube and primary peritoneal cancer prevention. NCI; 2016. [Full Text]

6. Suresh S, Sharma KK, Saksena M, Thukrak A, Agarwal R, Vatsa M. Predictors of breastfeeding problems in the first postnatal week and its effect on exclusive breastfeeding rate at six months: experience in a tertiary care centre in Northern India. Indian J Public Health 2014; 58(4): 270-3.

7. Bergmann RL, Bergmann KE, von Weizsacker K, Berns M, Henrich W, Dudenhausen JW. Breastfeeding is natural but not always easy: intervention for common medical problems of breastfeeding motehrs-a review of the scientific evidence. J Perinat Med 2014; 42(1): 9-18. [Full Text | DOI]
8. Hariprasar PG, Sheen LB, George B. Lactation problems in postnatal period: a hospital based study. Journal of Medical Science and Clinical Research 2017; 5(03): 19430-5. [Full Text $\mid \mathrm{DOI}]$

9. Odom EC, Li R, Scanlon KS, Perrine CG, Grummer-Strawn L. Reasons for earlier than desired cessation of breastfeeding. Journal of Pediatrics 2013; 131(3): e726-32. [Full Text | DOI]

10. National Statistics Bureau. Bhutan multiple indicator survey. NSB; 2011. [Full Text]

11. Kronborg H, Vaeth M. How are effective breastfeeding techniques and pacifiers use related to breastfeeding problems and breastfeeding duration? Birth 2009; 36(1): 3442. [Full Text | DOI]

12. Elsa RJG. Common problems during lactation and their management. Journal of Pediatrics 2004; 80(5): s147-54. [Full Text]

\section{AUTHORS CONTRIBUTION}

Following authors have made substantial contributions to the manuscript as under:

KZ: concept, analysis of data, study design, manuscript drafting and critical reviews

DW: concept, analysis of data, study design, manuscript drafting and revisions

TT: concept, analysis of data, study design, manuscript drafting and revisions

MSG: concept, analysis of data, study design, manuscript drafting and revisions

Authors agree to be accountable for all respects of the work in ensuring that questions related to the accuracy and integrity of any part of the work are appropriately investigated and resolved.

CONFLICT OF INTEREST

None

GRANT SUPPORT AND FINANCIAL DISCLOSURE

None 